\title{
Prevalence, Clinical Correlates, and Use of Glucose-Lowering Drugs among Older Patients with Type 2 Diabetes Living in Long-Term Care Facilities
}

\author{
Mario Bo, ${ }^{1}$ Stefano Gallo, ${ }^{1}$ Mauro Zanocchi, ${ }^{1}$ Paola Maina, ${ }^{2}$ \\ Luisa Balcet, ${ }^{3}$ Martina Bonetto, ${ }^{1}$ Lorenzo Marchese, ${ }^{1}$ \\ Annalisa Mastrapasqua, ${ }^{1}$ and Nicoletta Aimonino Ricauda ${ }^{1}$ \\ ${ }^{1}$ Department of Medical Sciences, Geriatric Section, University of Turin, 10124 Turin, Italy \\ ${ }^{2}$ ASL TO4, 10034 Piemonte, Italy \\ ${ }^{3}$ Ospedale Civico "Città di Settimo Torinese", 10036 Settimo Torinese, Italy \\ Correspondence should be addressed to Lorenzo Marchese; marcheselorenzo@virgilio.it
}

Received 10 September 2014; Accepted 27 November 2014

Academic Editor: Garth Warnock

Copyright (C) 2015 Mario Bo et al. This is an open access article distributed under the Creative Commons Attribution License, which permits unrestricted use, distribution, and reproduction in any medium, provided the original work is properly cited.

Prevalence, clinical correlates, and use of glucose-lowering drugs were comprehensively evaluated among 863 nursing home older patients with diabetes (mean age $82.9 \pm 2.1$ years): functional dependence and cognitive impairment were present in $84.1 \%$ and $68 \%$ of patients, respectively, and $66.3 \%$ of patients had $2-4$ comorbidities. HbAlc values $<7.0 \%$ were documented in $54.9 \%$ of diabetic; significantly lower HbAlc levels were observed in demented patients than in nondemented subjects. Documented hypoglycemic episodes were reported for 57 patients (6.6\%), without significant association with age, functional dependence, cognitive impairment, or HbAlc levels. About one-fifth of older long-term facilities residents have diabetes, with concomitant poor health conditions and high prevalence of cognitive impairment and functional dependence. Roughly three-fourths of these older and frail diabetic patients have HbAlc values lower than optimal, suggesting a potential for hypoglycemic harm especially among patients with severe cognitive impairment.

\section{Introduction}

There are few evidences about type 2 diabetes mellitus (T2DM) in frail elderly subjects and, specifically, in older patients living in long-term care residences [1-3]. These patients have usually reduced life expectancy, poor general health, and at least some degree of functional dependence and/or cognitive impairment. They represent those frail and vulnerable patients affected by T2DM for whom recent international guidelines specifically recommended less stringent glycemic targets and prioritized well-being and quality of life [4-6].

Duration of diabetes and advancing age independently predict morbidity and mortality rates in elderly subjects. Recent observations, demonstrating that cardiovascular complications and hypoglycemia are common among older diabetic patients, support the reorientation of care of older patients with T2DM away from intensive glycemic control as the core focus of management [7]. Target goal for glycated hemoglobin (HbAlc) in older adults generally should be $7.5 \%$ to $8 \%$. Although $\mathrm{HbAlc}$ between $7 \%$ and $7.5 \%$ may be appropriate if it can be safely achieved in healthy older adults with few comorbidities and good functional status, higher HbAlc targets (8\%-9\%) are appropriate for older adults with multiple comorbidities, poor health, and limited life expectancy [4-6]. Moreover there is potential harm in lowering $\mathrm{HbAlc}$ to less than $6.5 \%$ in older adults with type $2 \mathrm{DM}[8]$.

Despite these recommendations for older vulnerable diabetic patients, there is little evidence on prevalence, clinical correlates, and treatment of T2DM in elderly patients living in long-term care facilities. In the present study we aimed to comprehensively evaluate prevalence, clinical correlates, and use of glucose-lowering drugs among nursing home older patients with diabetes. 


\section{Materials and Methods}

In this prospective observational study, patients living in 83 long-term care facilities in Piedmont, Northern Italy, were evaluated during the period of March-August 2013; all patients aged $>65$ and affected by diabetes were enrolled, without exclusion criteria.

Signed informed consent from patients or carer was obtained for all participants and the study was conducted according to the Recommendations Guiding Physicians in Biomedical Research Involving Human Subjects [9].

For all the patients the following information was recorded: identification, age, gender, and date of admission. Relevant conditions (as dementia, immobilization, and pressure sores) were also recorded. A thorough medical chart review was performed in order to ascertain, as far as possible, type and age of onset of diabetes, current hypoglycemic therapy, previous hypoglycemic episodes, and last available blood chemistries including serum glucose and glycated hemoglobin (HbAlc) levels and pre- and postprandial glucose levels. Total daily drug burden was also recorded.

Body mass index (BMI, according to the formula weight $(\mathrm{kg}) /$ height $\left.\left(\mathrm{m}^{2}\right)\right)$ was calculated and categorized in 4 classes (underweight: BMI < 18; normal weight: BMI 18-24.9; overweight: BMI 25-29.9; obesity: BMI $\geq 30$ ). Standardized scales were used for the evaluation of functional autonomy and cognitive status. Functional status was evaluated using the Activities of Daily Living (ADL) scale [10] that measures six functions relating to activities necessary for self-care, in each of which the patient can be described as autonomous or dependent; a score equal to or higher than 2 identifies functional dependence. Cognitive status was evaluated using the Mini-Mental State Examination [11], a questionnaire evaluating several cognitive domains; score between 19 and 24/30 identifies mild cognitive impairment, while score between 10 and 18/30 and score below 10/30 identify moderate and severe cognitive impairment, respectively.

The data, collected on preprinted standardized protocols and subsequently transferred to MS Excel (Microsoft Inc.), were analyzed using SPSS/PC+. A preliminary explorative analysis was performed on continuous variables to assess normal distribution (skewness and kurtosis). The frequency of dichotomous and categorical variables was calculated, as well as the average and the standard deviation (SD) of continuous variables. Variables with Gaussian distribution were analyzed using Student's $t$-test and analysis of variance; variables without Gaussian distribution were analyzed using Pearson's Chi-square test. Dichotomous variables were analyzed using Chi-square test. The ANOVA test was used to evaluate differences between groups.

\section{Results}

Among 5076 residents in 83 long-term facilities, 863 patients with diabetes $(17 \%$, mean age $82.9 \pm 2.1$ years) were identified (Table 1), with female patients being significantly older than male patients (mean age $83.9 \pm 1.9$ versus $80.7 \pm 2.2$ years, $P<0.05)$. Among patients affected by diabetes there was a significantly greater prevalence of women than of men (582
TABLE 1: Characteristics of diabetic patients living in long-term facilities.

\begin{tabular}{|c|c|}
\hline \multicolumn{2}{|l|}{ Age (years) } \\
\hline Total & $82.9 \pm 2.1$ \\
\hline Male & $80.7 \pm 2.2$ \\
\hline Female & $83.9 \pm 1.9$ \\
\hline Females & $582(67.4 \%)$ \\
\hline \multicolumn{2}{|l|}{ BMI } \\
\hline$\leq 18$ & $46(5.3 \%)$ \\
\hline $18-24.9$ & $382(44.3 \%)$ \\
\hline $25-29.9$ & $206(23.9 \%)$ \\
\hline$\geq 30$ & $98(11.4 \%)$ \\
\hline N.D. & $131(15.1 \%)$ \\
\hline \multicolumn{2}{|l|}{ Preprandial serum glucose } \\
\hline$\leq 70 \mathrm{mg} / \mathrm{dL}$ & $47(5.5 \%)$ \\
\hline $71-126 \mathrm{mg} / \mathrm{dL}$ & $398(46.1 \%)$ \\
\hline $127-180 \mathrm{mg} / \mathrm{dL}$ & $224(25.9 \%)$ \\
\hline$\geq 181 \mathrm{mg} / \mathrm{dL}$ & $153(17.7 \%)$ \\
\hline N.D. & $41(4.8 \%)$ \\
\hline \multicolumn{2}{|l|}{ HbAlc } \\
\hline$<7 \%$ & $354(54.9 \%)$ \\
\hline $7-8.5 \%$ & $131(20.4 \%)$ \\
\hline$>8.5 \%$ & $159(24.7 \%)$ \\
\hline \multicolumn{2}{|l|}{ Functional dependence } \\
\hline Partial $(\mathrm{ADL}=1)$ & $435(50.4 \%)$ \\
\hline Total $(\mathrm{ADL} \geq 2)$ & $291(33.7 \%)$ \\
\hline $\begin{array}{l}\text { Moderate-severe cognitive impairment } \\
(\text { MMSE } \leq 18)\end{array}$ & $616(71.4 \%)$ \\
\hline \multicolumn{2}{|l|}{ Comorbidities } \\
\hline 0 & $7(0.8 \%)$ \\
\hline $1-2$ & $261(30.3 \%)$ \\
\hline $3-4$ & $388(44.9 \%)$ \\
\hline$\geq 5$ & $207(24.0 \%)$ \\
\hline
\end{tabular}

women versus 281 men, $67.4 \%$ and $32.6 \%$ of diabetic patients, resp.) and this difference was statistically significant among patients aged 80 or more $(P=0.000)$. More than $97 \%$ of patients were ascertained to be affected by T2DM. Clinical documentation about age of onset of DM was retrievable only in $25.3 \%$ of patients: mean duration of T2DM in these patients was $12.9 \pm 2.1$ years at the moment of observation.

It was possible to measure height and weight in 732 patients: roughly half of older diabetic patients were normal or underweight $(44.3 \%$ and $5.3 \%$, resp.), while a condition of overweight and obesity was documented in $23.9 \%$ and $11.4 \%$ of patients, respectively. Functional autonomy was documented in $15.9 \%$ of older diabetic patients, whereas partial or complete functional dependence was present in $50.4 \%$ and $33.7 \%$ of patients, respectively; $9.2 \%$ of patients were bedridden. Cognitive impairment was documented in $68 \%$ of diabetic patients, which was graded mild, moderate, and severe in $28.6 \%, 40.1 \%$, and $31.3 \%$ of them, respectively. Coexistence of some degree of functional dependence and 


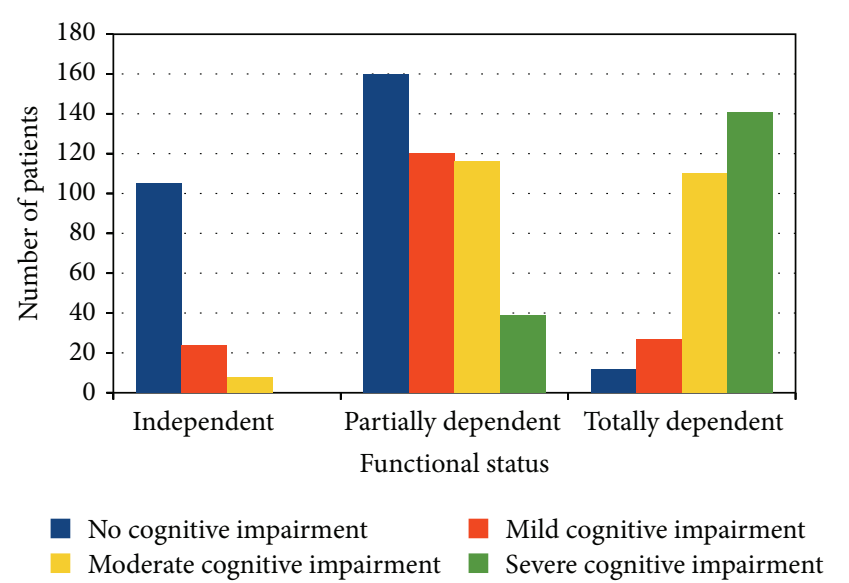

FIGURE 1: Distribution of cognitive impairment according to functional class among older diabetic patients.

cognitive impairment was documented in $95.9 \%$ of patients (Figure 1). At least one comorbidity was observed in $99.2 \%$ of patients, with $66.3 \%$ of patients having $2-4$ severe comorbidities. Hypertension (61.9\%), dementia (36.8\%), history of cardio- (22.8\%) and cerebrovascular (21.3\%) events, and bone fractures (13.4\%) were the most common comorbidities observed.

At the moment of data collection, $14.4 \%$ of diabetic patients were not receiving hypoglycemic therapy, $41.3 \%$ were treated with oral hypoglycemic drugs, $35.3 \%$ were receiving insulin therapy, and $9.0 \%$ received combination therapy with oral hypoglycemic drugs and insulin. Among patients treated with oral hypoglycemic drugs, metformin was used by $61.5 \%$, sulphonylureas by $33 \%$, repaglinide by $9.3 \%$, and a combination of metformin and glibenclamide by $4.8 \%$; the remaining patients were treated with metformin and pioglitazone $(0.8 \%)$, acarbose $(0.8 \%)$, and metformin and DPP4 inhibitors (0.6\%).

Among patients treated with insulin, 239 used rapidacting insulin (25.4\% human and $74.6 \%$ analogue), 58 were treated with intermediate-acting insulin, and 219 used longacting (glargine or detemir) insulin (90.4\% and 9.6\%, resp.). Among patients treated with insulin therapy, $45.4 \%$ received 4 doses a day and $33.1 \%$ received 3 doses daily.

At least one value of glycated hemoglobin (HbAlc) was available in $74.6 \%$ of patients: $54.9 \%$ of patients had HbAlc values < $7.0 \%, 20.4 \%$ had HbAlc values between $7.0 \%$ and $8.5 \%$, and $24.1 \%$ of patients had HbAlc values above $8.5 \%$. In the total sample of older diabetic patients, no significant association was observed between classes of HbAlc level $(<7.0 \%, 7.0-8.5 \%$, and $>8.5 \%)$ and either functional dependence or presence and severity of cognitive impairment, as well as mean HbAlc values, did not significantly differ in patients with or without functional dependence and cognitive impairment. Significantly lower HbAlc levels were observed in demented patients than in nondemented subjects (6.92 \pm $1.28 \%$ versus $7.23 \pm 1.67 \%, P=0.013)$.

Preprandial glycemic values (available for $95.2 \%$ of patients) below $126 \mathrm{mg} / \mathrm{dL}$ were documented in $51.6 \%$ of patients; $26 \%$ of patients had values between 126 and $180 \mathrm{mg} / \mathrm{dL}$ and $17.7 \%$ had values above $180 \mathrm{mg} / \mathrm{dL}$. Postprandial glycemic values under $180 \mathrm{mg} / \mathrm{dL}$ were observed in $37.2 \%$ of patients, $18.1 \%$ of patients had values between 181 and $250 \mathrm{mg} / \mathrm{dL}$, and $8.1 \%$ of patients had values above $250 \mathrm{mg} / \mathrm{dL}$. For $36.6 \%$ of patients there were no data available for postprandial glycemic values.

Documented hypoglycemic episodes were reported in medical charts for $57(6.6 \%)$ patients. At the moment of data collection, 30 of these patients $(52.6 \%)$ were treated with insulin, $22(38.5 \%)$ received oral hypoglycemic agents (9 received metformin and glibenclamide, 2 received metformin and other sulphonylureas, 5 received metformin, 5 received sulphonylureas, and 1 received metformin and repaglinide), and $3(5.2 \%)$ were treated with insulin and oral hypoglycemic agents ( 2 with acarbose and 1 with glibenclamide); 2 of these patients $(3.5 \%)$ were not receiving drugs at the moment of data collection.

Among patients with reported previous hypoglycemic episodes 11 (19.3\%) were bedridden and 19 (33.3\%) were affected by severe cognitive impairment. At the moment of data collection, previous hypoglycemic episodes were not associated with age, functional dependence, or cognitive impairment, although a trend to a greater prevalence of hypoglycemia among demented patients was observed. Mean HbAlc levels were not significantly lower in patients with previous reported hypoglycemia than in other diabetic patients $(6.83 \pm 1.18$ versus $7.143 \pm 1.37, P=0.08)$.

Single therapy with metformin was significantly more prevalent among patients without reported hypoglycemic episodes $(P=0.033)$. We did not observe significant association between use of sulphonylureas and hypoglycemic episodes, but therapy with metformin and glibenclamide was significantly more prevalent among patients with reported previous hypoglycemic episodes $(P=0.004)$. Patients receiving insulin therapy were significantly more prevalent among those with previous reported hypoglycemia $(P=0.009)$.

\section{Discussion}

We aimed to investigate prevalence, clinical correlates, and use of glucose-lowering drugs among older patients with T2DM living in long-term facilities in Piedmont, Northern Italy. Our study demonstrated that T2DM is a common clinical problem among these patients, affecting roughly less than one-fifth of residents, with a greater prevalence of the disease in women than in men. Extremely poor health conditions were documented in these patients. Less than one-fifth of them were functionally independent and roughly two-thirds of them had some degree of cognitive impairment, with concomitant functional dependence and cognitive impairment in more than $95 \%$ of patients. Two-thirds of patients had 2-4 severe comorbidities, mainly hypertension, dementia, cardioand cerebrovascular disease, and bone fractures. Finally, we documented a high prevalence of low HbAlc values and a remarkable incidence of documented hypoglycemic episodes among these cognitively and functionally impaired older residents. 
There are very few studies which investigated this item in similar populations. The prevalence of T2DM observed in our sample was in accordance with that observed in a French study, which reported a prevalence of T2DM of 17.1\% among 6275 older long-term facility residents aged 86 years [12]. Because mean duration of T2DM in the patients enrolled in our study was around 12 years, it is reasonable to suppose that diabetes onset in most of the patients occurred probably at an older age.

Despite current recommendations on hypoglycemic treatment and $\mathrm{HbAlc}$ targets for older, frail, and vulnerable patients [4-6] we found that more than half of long-term facility older residents had $\mathrm{HbAlc}$ values below 7\%, and 75\% of them had levels below $8.5 \%$. These findings demonstrate an undesired and potentially harmful aggressive hypoglycemic therapeutic approach in these frail and vulnerable patients. There are very few evidences about overall health benefit of hypoglycemic therapy among these frail patients, who are more vulnerable to and at higher risk of incident hypoglycemic episodes [8]. Therefore, recent international guidelines support the reorientation of care of older patients with T2DM away from intensive glycemic control as the core focus of management [7], and HbAlc targets around 8\%$9 \%$ are deemed appropriate for older adults with multiple comorbidities, poor health, and limited life expectancy [46]. Moreover, HbAlc levels were significantly lower among demented patients, who are more prone to the negative consequences of hypoglycemia. There is a burden of evidence linking hypoglycemia and cognitive decline: cognitively impaired and demented patients are more prone and vulnerable to hypoglycemia, which itself represents a major risk factor for further cognitive decline [13-18]. Unawareness of hypoglycemia and subtle or atypical clinical presentation make extremely difficult an early diagnosis of hypoglycemia in demented, frail patients, leading to the potential for major harm in these patients.

Hypoglycemic episodes were reported in medical charts in $6.6 \%$ of patients, probably underestimating the true prevalence of this feared complication. Among patients with reported hypoglycemic events, roughly one-fifth of them were bedridden and one-third had severe cognitive impairment. At the moment of data collection HbAlc values below $7.5 \%$ were yet more frequent in patients with previous hypoglycemic episodes than in patients without prior hypoglycemic episodes (75.4\% versus $45.1 \%, P=0.03)$. Combination therapy with metformin and glibenclamide and insulin therapy were both significantly more frequent among patients with previous hypoglycemic episodes, whereas single therapy with metformin was more prevalent among T2DM patients without previous hypoglycemic episodes. These findings are in keeping with and reinforce current Beers recommendations about potentially inappropriate medication use in the elderly: metformin is considered the safest oral glucose-lowering approach in diabetic patients without specific contraindications, whereas rapid-acting insulins are considered the drugs with the highest potential for harmful hypoglycemic events [19]. However, because of the crosssectional retrospective medical charts study, these findings should be carefully considered because we were able to document current glucose-lowering drug therapy at the moment of collection of data but we could not ascertain from medical charts which therapies were administered at the moment of hypoglycemic crisis.

Some limitations of the present study should be considered. The main limitation is inherent to the retrospective design of the study, based on data extracted from longterm facilities medical charts not scrupulously filled in. Moreover, retrospective observation makes it extremely difficult to define causality between adverse events and current hypoglycemic therapy, which however was not among the main goals of this study. On the other hand, this study in our view has some strengths that should be highlighted. To the best of our knowledge this is one of the first attempts to comprehensively evaluate global health status, including functional and cognitive conditions, among older patients with T2DM in long-term facilities. The high number of patients enrolled from a variegated sample of regional longterm facilities and the close similarity of our findings with results from the French study suggest that our results may reasonably and wisely be generalized to older Southern Europe patients living in long-term facilities.

In conclusion, our results documented that roughly three-fourths of older and frail diabetic patients living in long-term residences have $\mathrm{HbAlc}$ values lower than optimal, suggesting a potential for hypoglycemic harm especially among patients with severe cognitive impairment. Despite the current recommendations that strongly advise using "soft" HbAlc targets and wise and safe glucose-lowering medical therapies in these vulnerable patients, our findings seem to suggest an inappropriate and aggressive glucoselowering therapeutic approach in most of these frail and vulnerable elderly residents.

\section{Conflict of Interests}

The authors declare that there is no conflict of interests regarding the publication of this paper.

\section{References}

[1] E. Selvin, J. Coresh, and F. L. Brancati, "The burden and treatment of diabetes in elderly individuals in the U.S.", Diabetes Care, vol. 29, no. 11, pp. 2415-2419, 2006.

[2] G. S. Meneilly, "Diabetes in the elderly," Medical Clinics of North America, vol. 90, no. 5, pp. 909-923, 2006.

[3] K. H. Kahler, M. Rajan, G. G. Rhoads et al., "Impact of oral antihyperglycemic therapy on all-cause mortality among patients with diabetes in the veterans health administration," Diabetes Care, vol. 30, no. 7, pp. 1689-1693, 2007.

[4] A. F. Brown, C. M. Mangione, D. Saliba, and C. A. Sarkisian, "California Healthcare Foundation/American Geriatrics Society Panel on improving care for elders with Diabetes. Guidelines for improving the care of the older persons with diabetes mellitus," Journal of the American Geriatrics Society, vol. 51, pp. S265-S280, 2003.

[5] F. Ismail-Beigi, "Glycemic management of type 2 diabetes mellitus," The New England Journal of Medicine, vol. 366, no. 14, pp. 1319-1327, 2012. 
[6] American Geriatrics Society Expert Panel on the Care of Older Adults with Diabetes Mellitus, "Guidelines abstracted from the American Geriatrics Society Guidelines for improving the care of older adults with diabetes mellitus: 2013 update," Journal of the American Geriatrics Society, vol. 61, no. 11, pp. 2020-2026, 2013.

[7] E. S. Huang, N. Laiteerapong, J. Y. Liu, P. M. John, H. H. Moffet, and A. J. Karter, "Rates of complications and mortality in older patients with diabetes mellitus: the diabetes and aging study," JAMA Internal Medicine, vol. 174, no. 2, pp. 251-258, 2014.

[8] S. E. Inzucchi, R. M. Bergenstal, J. B. Buse et al., "Management of hyperglycemia in type 2 diabetes: a patient-centered approach: position statement of the American Diabetes Association (ADA) and the European Association for the Study of Diabetes (EASD).," Diabetes care, vol. 35, no. 6, pp. 1364-1379, 2012.

[9] World Medical Association Declaration of Helsinki, "Recommendations guiding physicians in biomedical research involving human subjects," Cardiovascular Research, vol. 35, pp. 2-3, 1997.

[10] S. Katz, T. D. Downs, H. R. Cash, and R. C. Grotz, "Progress in development of the index of ADL.," Gerontologist, vol. 10, no. 1, pp. 20-30, 1970.

[11] R. M. Crum, J. C. Anthony, S. S. Bassett, and M. F. Folstein, "Population-based norms for the mini-mental state examination by age and educational level," The Journal of the American Medical Association, vol. 269, no. 18, pp. 2386-2391, 1993.

[12] P. D. Barreto, C. Sanz, B. Vellas, M. Lapeyre-Mestre, and Y. Rolland, "Drug treatment for diabetes in nursing home residents," Diabetic Medicine, vol. 31, no. 5, pp. 570-576, 2014.

[13] J. S. Saczynski, S. Siggurdsson, P. V. Jonsson et al., "Glycemic status and brain injury in older individuals: the age gene/environment susceptibility-Reykjavik study," Diabetes Care, vol. 32, no. 9, pp. 1608-1613, 2009.

[14] R. A. Whitmer, A. J. Karter, K. Yaffe, C. P. Quesenberry Jr., and J. V. Selby, "Hypoglycemic episodes and risk of dementia in older patients with type 2 diabetes mellitus," The Journal of the American Medical Association, vol. 301, no. 15, pp. 1565-1572, 2009.

[15] J. M. Nelson, K. Dufraux, and P. F. Cook, “The relationship between glycemic control and falls in older adults," Journal of the American Geriatrics Society, vol. 55, no. 12, pp. 2041-2044, 2007.

[16] I. Feinkohl, P. P. Aung, M. Keller et al., "Severe hypoglycemia and cognitive decline in older people with type 2 diabetes: the edinburgh type 2 diabetes study," Diabetes Care, vol. 37, no. 2, pp. 507-515, 2014.

[17] N. N. Zammitt and B. M. Frier, "Hypoglycemia in type 2 diabetes: pathophysiology, frequency, and effects of different treatment modalities," Diabetes Care, vol. 28, no. 12, pp. 29482961, 2005.

[18] J. P. Bremer, K. Jauch-Chara, M. Hallschmid, S. Schmid, and B. Schultes, "Hypoglycemia unawareness in older compared with middle-aged patients with type 2 diabetes," Diabetes Care, vol. 32, no. 8, pp. 1513-1517, 2009.

[19] The American Geriatrics Society 2012 Beers Criteria Update Expert Panel, "American Geriatrics Society updated Beers Criteria for potentially inappropriate medication use in older adults," Journal of the American Geriatrics Society, vol. 60, no. 4, pp. 616-631, 2012. 


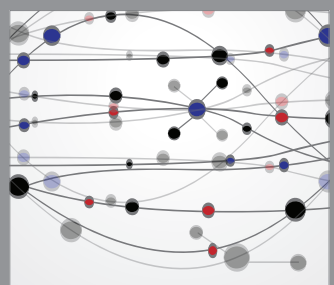

The Scientific World Journal
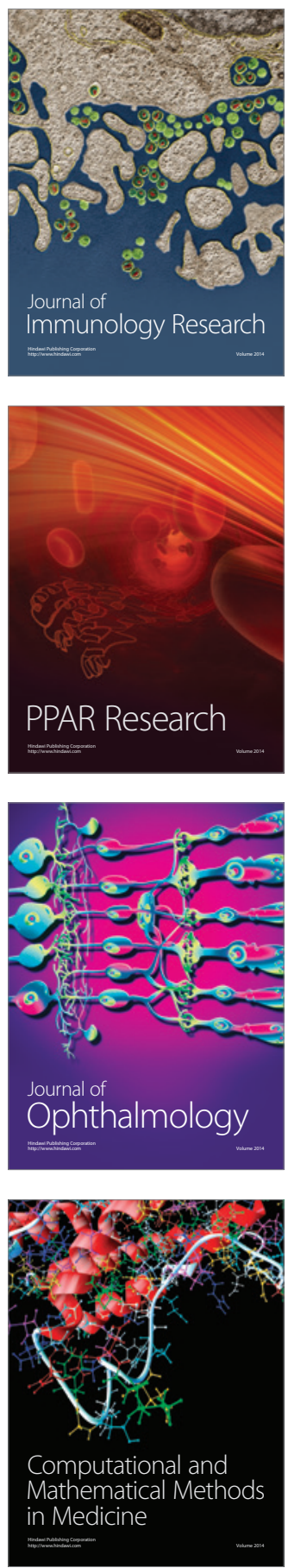

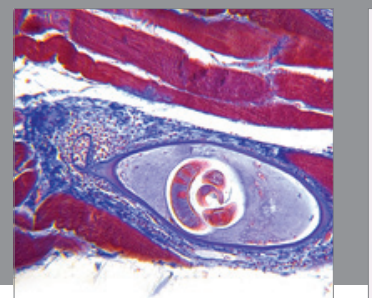

Gastroenterology

Research and Practice
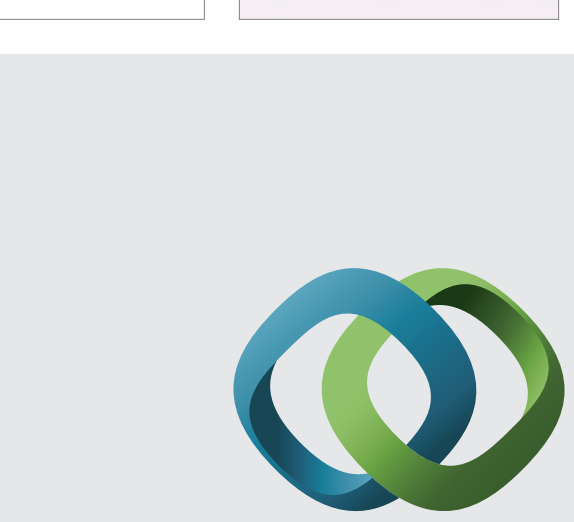

\section{Hindawi}

Submit your manuscripts at

http://www.hindawi.com
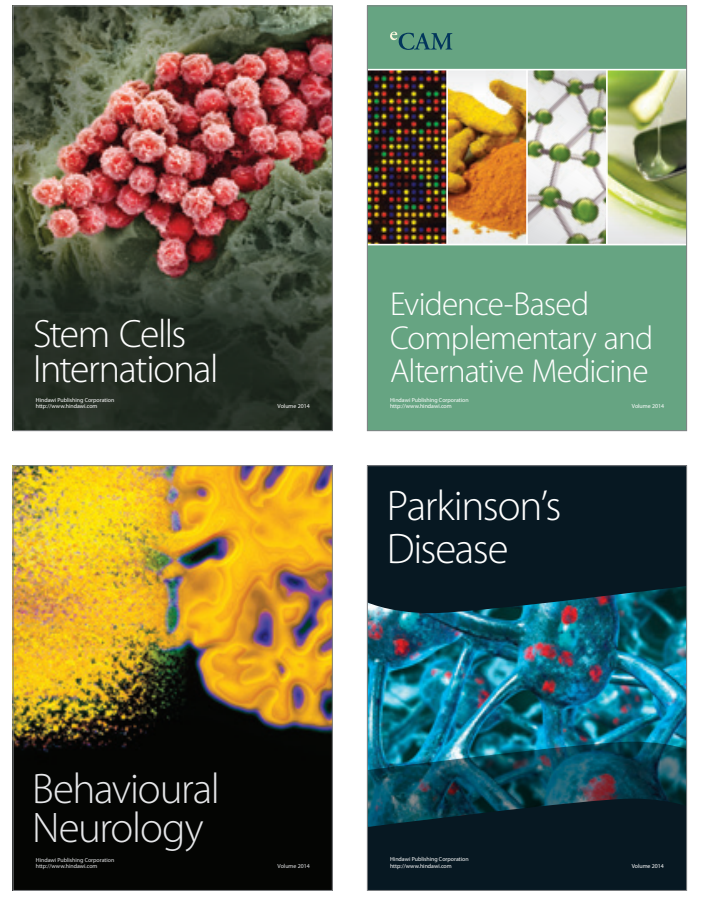
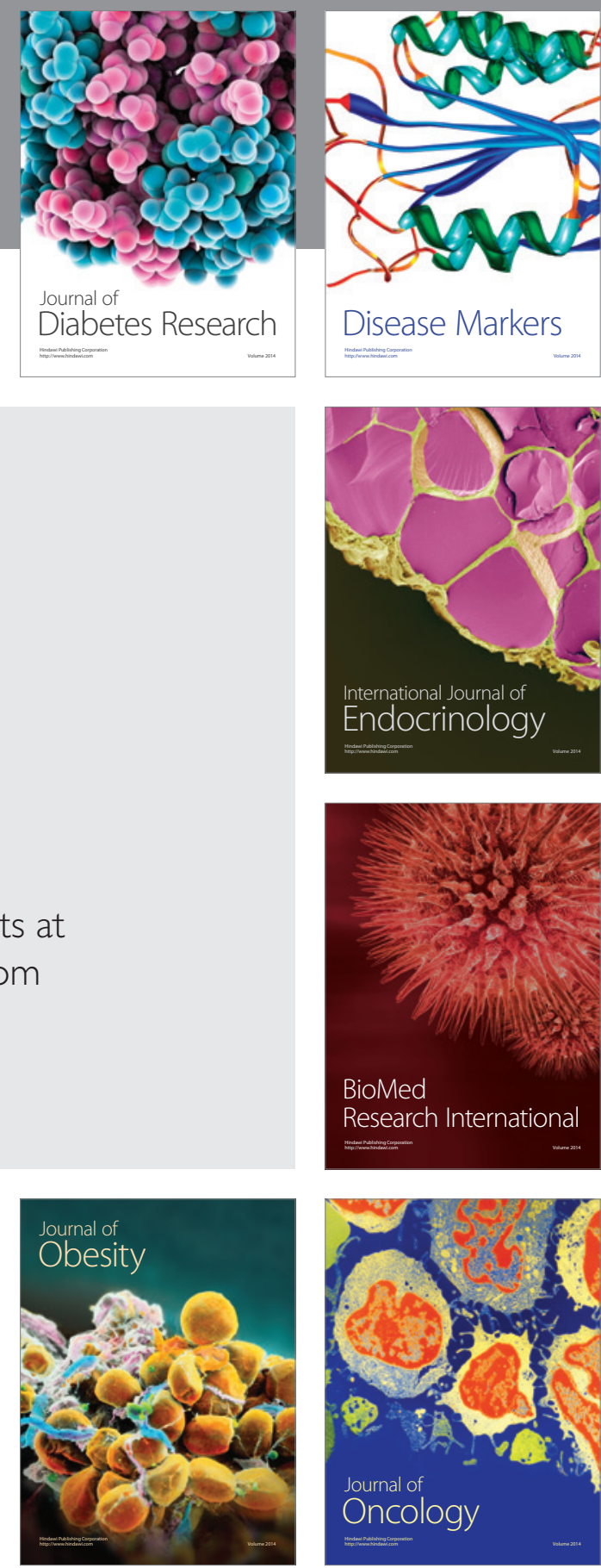

Disease Markers
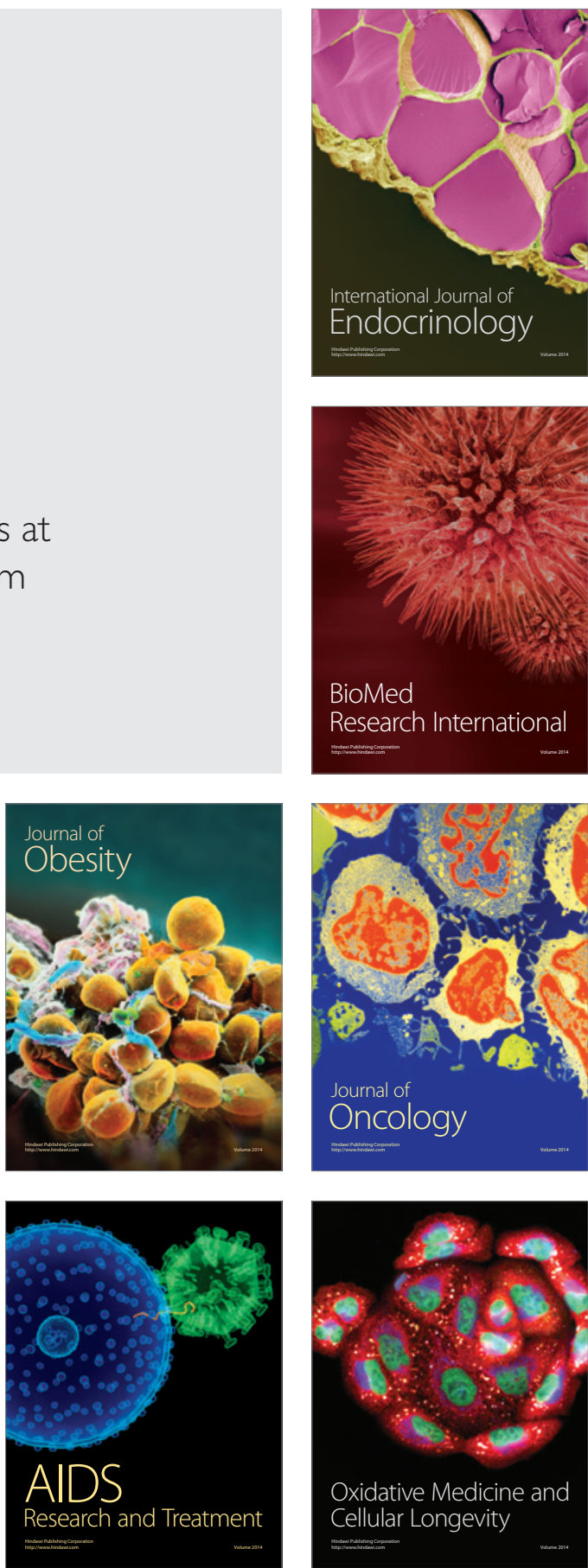